\title{
Aplicación de un calendario de pruebas de evaluación continua en la asignatura "Obstetricia" en sexto de Medicina
}

\section{Application of a continuous assessment tests in the subject "Obstetrics" in the sixth year of Medicine}

\section{Carlos S. Piñel Pérez ${ }^{1 *}$, María José Gómez-Roso Jareño ${ }^{2}$ y Juan José López Galián ${ }^{3}$}

1 Servicio de Obstetricia y Ginecología del Hospital Quirónsalud San José (Madrid). Profesor Asociado de la Universidad Europea (Madrid); carlos.s.pinel@gmail.com, ORCID ID: https://orcid.org/0000-0001-6647-8718

2 Servicio de Obstetricia y Ginecología del Hospital Quirónsalud San José (Madrid). Profesora colaboradora de la Universidad Europea (Madrid); mariajose.grj@gmail.com

3 Servicio de Obstetricia y Ginecología del Hospital Quirónsalud San José (Madrid). Profesor Asociado de la Universidad Europea (Madrid); jlopezgalian@yahoo.es

* Correspondencia: carlos.s.pinel@gmail.com

Recibido: 30/9/2020; Aceptado: 14/10/2020; Publicado: 18/10/2020

Resumen: Introducción. Con el objetivo de promover y motivar al aprendizaje continuo durante el curso de la asignatura de Obstetricia (6º Medicina), se diseñó un calendario de "Pruebas de Evaluación Continua" (PEC). Este aprendizaje progresivo permite interiorizar conceptos, mejor seguimiento de las clases sucesivas y facilita el estudio, mejorando el rendimiento final. Métodos. Se diseñaron 4 PEC voluntarias, una por bloque del temario, con 10 preguntas tipo test sin puntuación negativa por error, para estimular al alumnado a pensar en cada una de las cuestiones y tomar decisiones. Las PEC se realizaron en los últimos 10 minutos de la última clase de cada bloque. Medicina tiene cinco grupos, uno por hospital docente. Esta iniciativa se realizó sólo en uno de los grupos (grupo A). Resultados. Se presentaron a las PEC el 90,9\% de los alumnos matriculados. En la primera PEC la calificación media fue 68\% (máximo 90\%; mínimo 50\%; moda 70\%), en la segunda 88\% (máximo 100\%; mínimo 60\%; moda 90\%), en la tercera 95\% (máximo 100\%; mínimo 80\%; moda 100\%) y en la cuarta $99 \%$ (máximo 100\%; mínimo 90\%, moda 100\%). La calificación media en el examen final de la asignatura (común a los 5 grupos) del grupo A fue de 7,300 (sobre 10), superior a la de los grupos B $(6,886), C(6,592), D$ $(6,572)$ y E $(6,463)$. Conclusiones. Se objetivó un aumento del rendimiento progresivo grupal e individual. Los resultados de la primera PEC fueron más dispersos, y posteriormente se fueron homogeneizando. El grupo en el que se realizaron las PEC obtuvo mejor resultado en el examen final que los otros grupos.

Palabras clave: evaluación continua; evaluación; aprendizaje continuo

Abstract: Introduction. We designed a "Continuous Assessment Tests" (CAT) for promoting and motivating continuous learning during 
the course of the subject "Obstetrics" (6th year of Medicine). This progressive learning enables concepts to be internalized, better monitoring of successive classes and facilitates study, improving results. Methods. Four voluntary CAT were designed, one per block of the subject, with 10 multiple choice questions without negative score by mistake to stimulate students to think about each of the questions and make decisions. The CAT were performed in the last 10 minutes of the last class of each block. Medicine has five groups, one per teaching hospital. This initiative was carried out in only one of the groups (group A). Results. 90.9\% of the enrolled students attended the CAT. In the first CAT the mean score was 68\% (maximum 90\%; minimum $50 \%$; mode $70 \%$ ), in the second $88 \%$ (maximum $100 \%$; minimum $60 \%$; mode $90 \%$ ), in the third 95\% (maximum 100\%; minimum $80 \%$; mode $100 \%$ ) and in the fourth 99\% (maximum 100\%; minimum $90 \%$, mode $100 \%$ ). The mean score in the final exam of the subject (common to the 5 groups) of group A was 7,300 (out of 10), higher than that of groups B $(6,886), C(6,592), D(6,572)$ and $E(6,463)$. Conclusions. An increase in group and individual progressive yield was observed. The results of the first CAT were more dispersed, and later they were homogenized. The group in which the CAT were performed got better yield in the final exam than the other groups.

Key words: continuous assessment; assessment; continuous learning

\section{Introducción}

Son varios los trabajos previos que avalan el uso de la evaluación continua en la enseñanza universitaria, demostrando una mejora en los resultados académicos y en el número de alumnos presentados (1). De esta forma, se invita al estudiante a estudiar de una manera más progresiva y evitar un estudio final dirigido exclusivamente al examen, un modelo de estudio habitual cuando la evaluación es final y única, pero en el que se dedica menos tiempo del necesario y mediante el cual se propicia un estudio que aporta un aprendizaje puntual, superficial y selectivo del contenido de la materia (2). Aparte de esta mejora de rendimiento y de aprendizaje, hay estudios que añaden que la evaluación continua es positiva para la motivación del estudiante de Medicina (3).

El curso académico 2018/2019 fue el primero en el que hubo alumnos en Sexto de Medicina en el Complejo Hospitalario Rúber Juan Bravo / San José, uno de los 5 hospitales docentes de la Universidad Europea en Madrid. Si bien durante el curso se objetivó un gran interés sobre la asignatura de Obstetricia por gran parte del alumnado, en el examen final se encontraron unos resultados inferiores a los esperados. Uno de los motivos a los que achacamos este rendimiento por debajo de lo esperado, fue la forma de estudiar de los alumnos. El examen de nuestra asignatura es el último del cuatrimestre, y el alumnado tiende a hacer un estudio "para el examen", dedicándole pocos días al estudio profundo de la asignatura y por tanto obteniendo un rendimiento pobre en el examen. Durante el curso 2019/2020 decidimos implementar un calendario de pruebas de evaluación continua con el objetivo de 
promover y motivar al aprendizaje continuo durante todo el curso. Nuestra hipótesis era que de este modo los estudiantes lograrían un aprendizaje progresivo que les permitiría interiorizar conceptos, conseguir un mejor seguimiento de las clases sucesivas y les facilitaría el estudio, mejorando el rendimiento final.

\section{Métodos}

Se diseñaron cuatro pruebas de evaluación continua. Cada una de ellas correspondió a uno de los bloques del temario y se llevaron a cabo en los últimos 10 minutos de clase de la última clase de cada bloque teórico. Las pruebas consistieron en 10 preguntas de opción múltiple, con una única respuesta correcta. No se asignó puntuación negativa al error, con el objetivo de estimular a los alumnos a pensar en la cuestión y tomar decisiones, sin miedo a la penalización por el error y, por tanto, al abandono precoz de la pregunta sin pensar en su contenido y sin arriesgarse a decidir. Estas pruebas de evaluación continua fueron de realización voluntaria y no fueron "eliminatorias" de materia. La influencia de su resultado en la calificación final consistía en ser el elemento decisorio en caso de optar a matrícula de honor, de manera que, si los alumnos obtenían más de un 70\% de rendimiento medio en las pruebas de evaluación continua, podían aspirar a la matrícula en caso de una elevada calificación en el examen final. Inmediatamente tras la realización de las pruebas, se realizó la corrección de cada una de las preguntas con las soluciones y la explicación de cada una de ellas.

Esta experiencia concreta se realizó en el Grupo del Complejo Hospitalario Rúber Juan Bravo / San José. El examen final teórico de la asignatura es común a los cinco grupos de Sexto de Medicina de la Universidad Europea en Madrid. Para ese estudio hemos denominado grupo A al grupo al que se le aplicó el calendario de pruebas de evaluación continua y grupos B, C, D y E a los demás grupos. El examen final teórico, común a todos los grupos y simultáneo, consistió en una prueba de 60 preguntas de opción múltiple, con una única respuesta correcta y con calificación negativa del error.

Se analizó para este estudio la participación de los alumnos del grupo A en las pruebas de evaluación continua, sus resultados en las mismas y el rendimiento final en el examen final teórico.

\section{Resultados}

\subsection{Participación y resultados en las Pruebas de Evaluación Continua}

La participación en las pruebas de evaluación continua por parte de los alumnos del grupo A fue del 90,9\% (Figura 1). El rendimiento de los alumnos en las pruebas de evaluación continua fue mejorando y homogeneizándose progresivamente, como se puede ver en la Tabla 1 y en las Figuras 2 y 3, probablemente por un aumento progresivo de la motivación al estudio. 


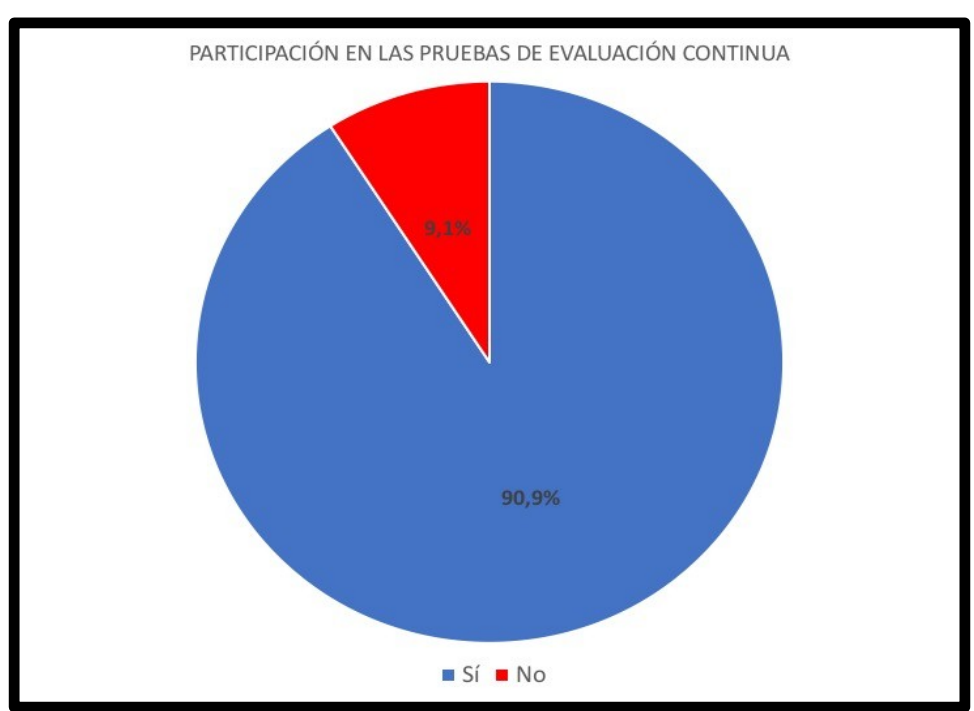

Figura 1. Participación de los estudiantes del Grupo A en las pruebas de evaluación continua

Tabla 1. Resultados de las Pruebas de Evaluación Continua

\begin{tabular}{ccccc}
\hline Calificación & PEC 1 & PEC 2 & PEC 3 & PEC 4 \\
\hline Media & $68 \%$ & $88 \%$ & $95 \%$ & $99 \%$ \\
Moda & $70 \%$ & $90 \%$ & $100 \%$ & $100 \%$ \\
Mínimo & $50 \%$ & $60 \%$ & $80 \%$ & $90 \%$ \\
Máximo & $90 \%$ & $100 \%$ & $100 \%$ & $100 \%$ \\
\hline
\end{tabular}

PEC: Prueba de Evaluación Continua

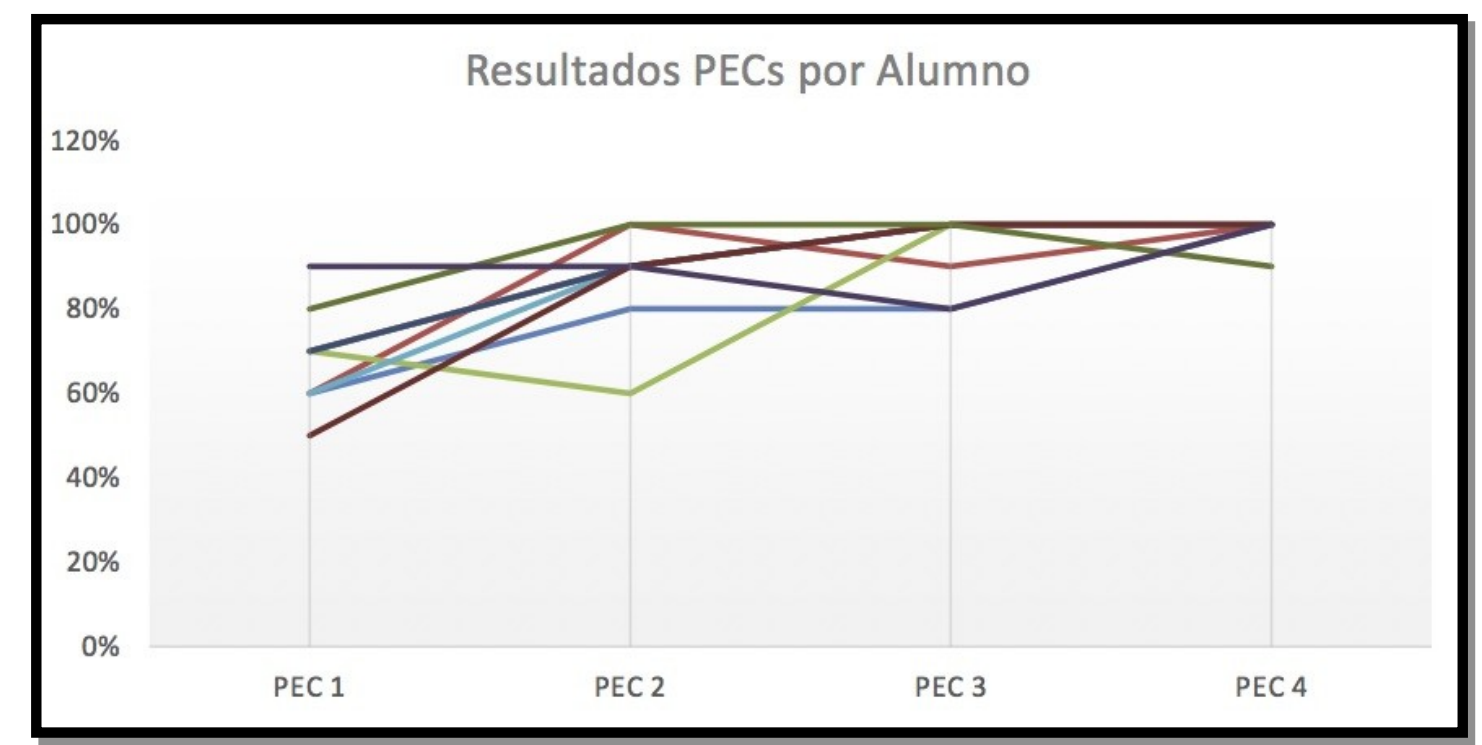

Figura 2. Resultados de las Pruebas de Evaluación Continua por Alumno 


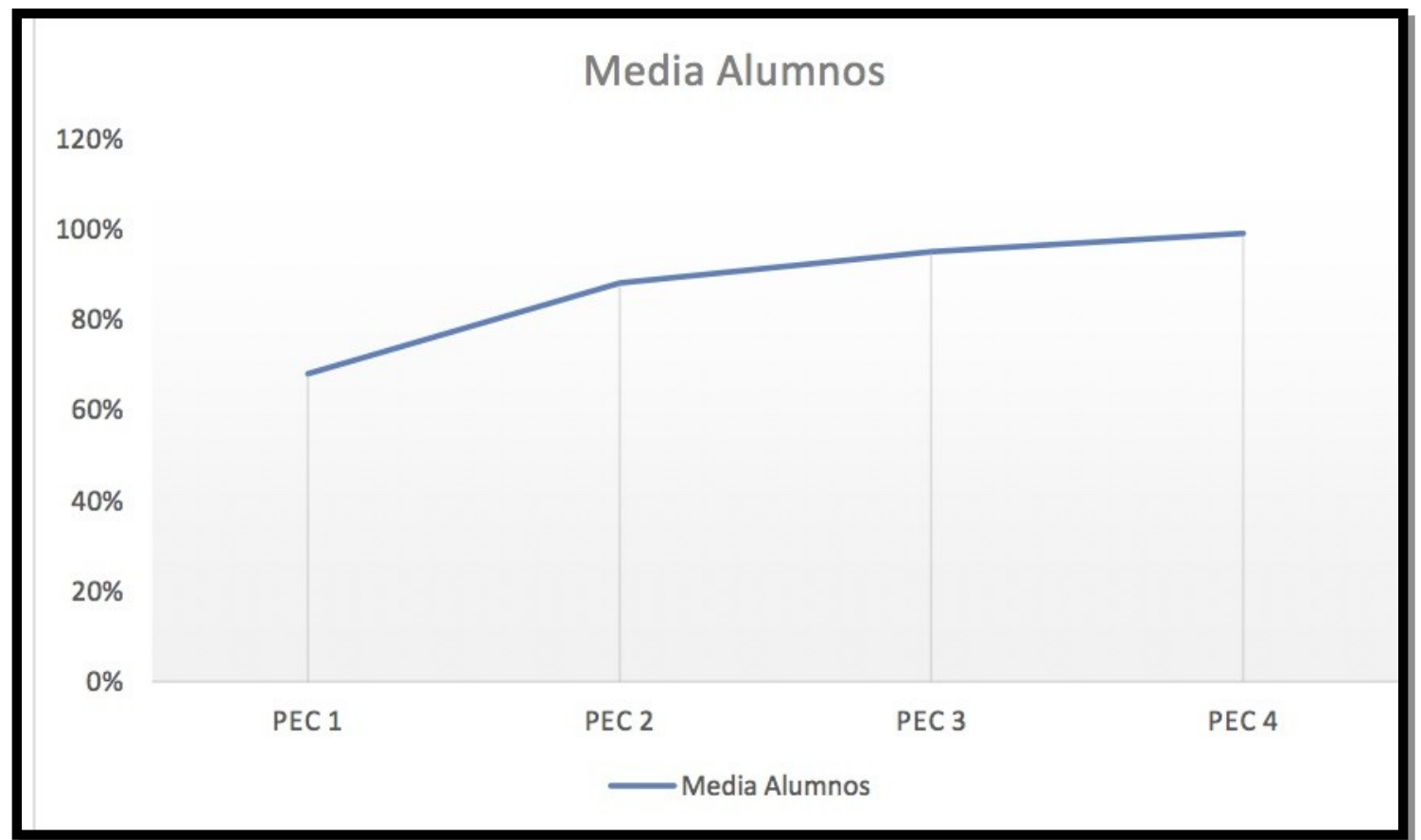

Figura 3. Media de la calificación obtenida en las Pruebas de Evaluación Continua por alumno

\subsection{Resultados en la calificación de la prueba teórica final}

Como se muestra en la Figura 4, se observó un mayor rendimiento de los alumnos del grupo A, ya que la calificación media en el examen teórico final de la asignatura (común a los 5 grupos) fue de 7,300 (sobre $10)$, superior a la de los grupos $B(6,886), C(6,592), D(6,572)$ y $E$ $(6,463)$.

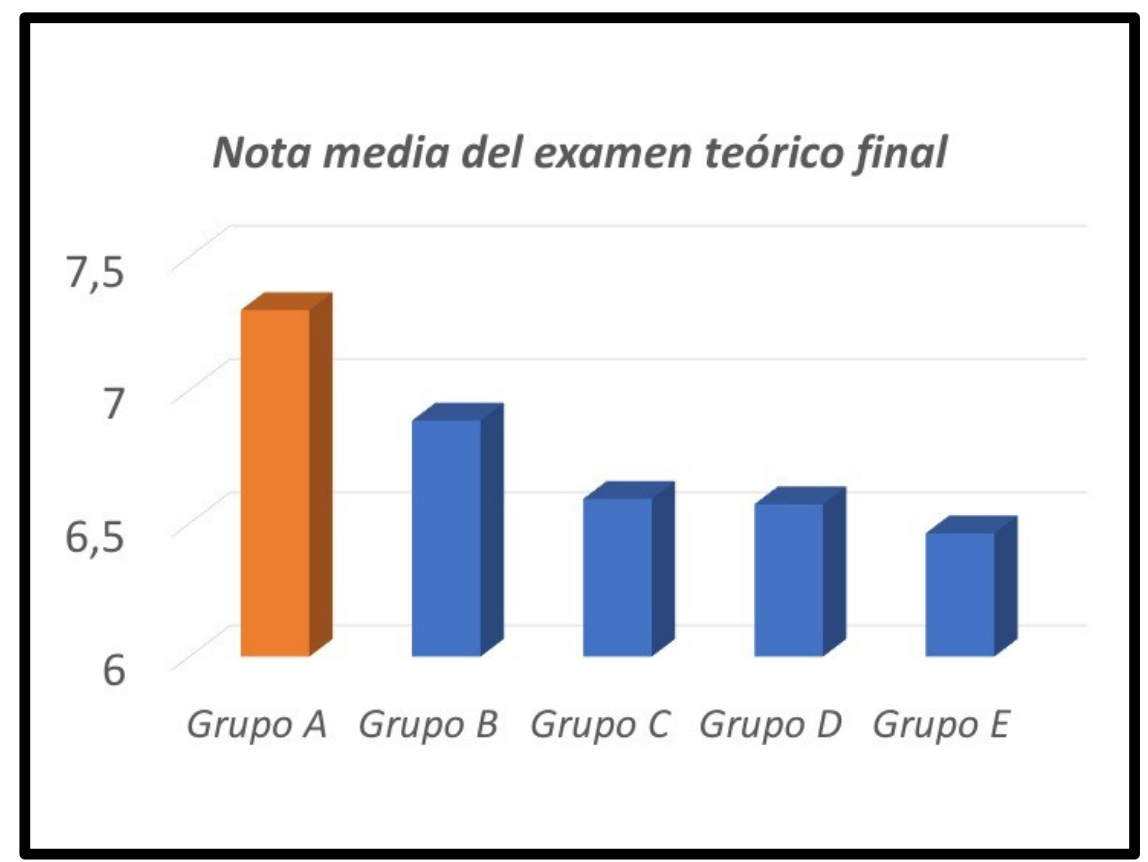

Figura 4. Calificación media en el examen teórico final de los alumnos de todos los grupos 


\section{Discusión}

Se eligió el formato de prueba de preguntas de elección múltiple por dos motivos. Por un lado, por ser la misma tipología de pregunta a la que se enfrentan en el examen final teórico de la asignatura, con lo que se persigue que la percepción del alumno frente a la prueba de evaluación continua sea que resulta útil como herramienta de entrenamiento. Además, este tipo de prueba es adecuada para evaluar resultados de conocimientos, comprensión, aplicación y análisis, como se persigue con esta medida $(4,5)$.

Pese a ser la primera vez que se adoptaba esta medida y a pesar del hecho de que no tuviese un peso específico en la evaluación final, el índice de participación, del 90,9\%, fue bastante alto. Probablemente, esto se debe al hecho de realizar la prueba en el aula, dentro del horario de clase.

En nuestra muestra se encontró un aumento progresivo del rendimiento y una homogenización paulatina de los resultados, como se ve en las Figuras 2 y 3 . En la primera PEC, se obtuvieron unos resultados pobres, con una media de tan sólo el 68\% de las respuestas acertadas. Muy pocos estudiantes obtuvieron calificaciones altas y ninguno hizo la prueba perfecta. Sin embargo, según avanza el curso y van realizando las demás PEC se objetiva un aumento paulatino del rendimiento, para, en la última prueba, encontrar que la gran mayoría de los alumnos realizan la prueba perfecta, sin errores. Los autores achacamos esta mejoría en el rendimiento a dos aspectos. En primer lugar, creemos que los resultados de la primera PEC les hizo ver que la preparación que estaban haciendo de la asignatura no era buena, lo cual les hizo estudiar más para la segunda. Al estudiar más, como se deriva de los resultados de la segunda PEC, no sólo obtienen mejores resultados, sino que aprecian que hacen un mejor seguimiento de las clases. Esto supone un doble refuerzo positivo al estudio: siguen mejor las clases y obtienen mejor resultado en la PEC, con lo que siguen estudiando, e incluso estudian con más ímpetu, y de este modo mejoran el aprendizaje y acaban obteniendo calificaciones excelentes en las últimas PEC. De manera subjetiva, el profesorado también advirtió un mayor interés y participación del alumnado en las clases. De este modo, como señalan Aftab y cols. (3), la evaluación continua actúa como herramienta motivacional para el estudiante. Nuestro grupo también ha identificado en el pasado (6) este efecto motivacional al poner a los estudiantes en situación de toma de decisiones mediante un concurso por equipos. En ambos casos, interpretamos que al enfrentarse a un problema y ver qué no pueden o no saben resolverlo con soltura, se motivan para el estudio de cara a estar mejor preparados para el examen, obteniendo un mayor aprendizaje del contenido.

Además, es reseñable la homogenización de los resultados. Al principio los resultados son más dispersos. Los estudiantes que en la primera PEC obtienen buenos resultados, siguen haciéndolo; pero los que obtienen peores calificaciones van mejorando hasta situarse en el 
mismo escalón de rendimiento, lo cual muestra que la aplicación de este calendario de pruebas de evaluación continua optimiza el rendimiento de los estudiantes que, sin la motivación extra, no se preparan igual durante el curso.

El objetivo final de esta iniciativa es mejorar el aprendizaje y el rendimiento final de los alumnos, lo cual se logró, a la luz de los resultados en el examen final teórico común a los otros grupos, donde los estudiantes a los que se les aplicó el calendario de pruebas de evaluación continua con este método descrito, obtuvieron una calificación media superior a los otros grupos, como se ve en la Figura 4. De este modo, se cumple la premisa vista en otros trabajos en los que la evaluación continua mejora los resultados académicos (1). En nuestro trabajo, además, se muestra que esta mejora no es debida a la "eliminación" de materia con las pruebas, ya que el examen final es el único que cuenta en la evaluación teórica y corresponde al contenido de toda la asignatura. Nosotros creemos que la mejora es debida al modo de estudio. Al estudiar progresivamente durante todo el curso, el aprendizaje es más profundo, más completo y mejor; lo cual se traduce en un mejor rendimiento en la prueba de evaluación final. De esta forma, evitamos ese estudio final dirigido exclusivamente al examen, y lo sustituimos por un aprendizaje continuo, que, indudablemente, deja más poso en el estudiante (2) y le permite afianzar conceptos, no sólo de cara a la evaluación final, si no como parte fundamental de su formación como médico.

Una de las limitaciones de esta experiencia es que no se evaluó la satisfacción del alumnado con la medida ni su efecto sobre el estrés, una condición asociada a peores resultados de rendimiento (7). Sería interesante en el futuro investigar sobre cómo percibe el alumnado estas pruebas. Si aumentan su estrés por verse sometidos a pruebas de evaluación periódicas o si, por el contrario, el hecho de afianzar conceptos les da seguridad de cara a la evaluación final y, por tanto, les permite afrontar con mayor tranquilidad periodo de evaluaciones finales.

Para concluir, nuestra experiencia muestra que la evaluación continua, pese a no ser "eliminatoria", resulta motivadora para el alumnado, ya que le permite darse cuenta de la importancia del estudio continuo y de sus beneficios tanto para el seguimiento del resto de las clases como de su aprendizaje. Además, mejora el rendimiento académico final. De este modo, obtenemos un mejor resultado $\mathrm{y}$, probablemente, un mayor aprendizaje del contenido, que es el fin último de la docencia. Por tanto, creemos que la evaluación continua debe dejar de ser, como señalaban Gual y Palés-Argullós en 2012, una "rara avis" en Medicina (8).

\section{Conclusiones}

- Se objetivó un aumento del rendimiento progresivo grupal e individual. 
- Los resultados de la primera PEC fueron más dispersos y posteriormente se fueron homogeneizando.El grupo en el que se aplicó el calendario de pruebas de evaluación continua obtuvo mejor resultado en el examen final que los otros grupos.

- En futuras experiencias sería interesante investigar sobre cómo percibe el alumnado estas pruebas y su influencia sobre el estrés del estudiante.

Financiación: No ha habido financiación.

Declaración de conflicto of interés: Los autores declaran no tener ningún conflicto de intereses.

\section{Referencias}

1. Montolio D. The impact of continuous assessment on a temporal perspective: the results of a pioneering experiment at the University of Barcelona (Spain). Mult J Edu Soc \& Tec Sci, 2015, 2 (1), 128-140. http://doi.org/10.4995/muse.2015.3447

2. Ricoy MC, Fernández-Rodríguez J. La percepción que tienen los estudiantes universitarios sobre la evaluación: un estudio de caso. $\begin{array}{lllll}\text { Educación } & X X 1, \quad 2013, & 16 & \text { (2), }\end{array}$ http://doi.org/10.5944/educxx1.16.2.2645

3. Aftab MT, Tariq MH. Continuous assessment as a good motivational tool in medical education. Acta medica academica, 2018, 47 (1), 76-81. http://doi.org/10.5644/ama2006-124.216

4. Epstein RM. Assessment in medical education. $N$ Engl J Med, 2007, 356 (4), 387-396. http://doi.org/10.1056/NEJMra054784

5. Grivent M, Baillés E, Moyano E, Pérez J. Relación entre pruebas de elección múltiple y de ensayo en los grados de Biología Humana y Medicina de la Universitat Pompeu Fabra. FEM, 2019; 22(3), 109-113. URL:

https://www.educacionmedica.net/pdf/revista/2203/FEM 2203109 O 251 9011 Perez.pdf (último acceso 29/9/20).

6. Piñel Pérez CS, Gómez-Roso Jareño MJ, López Galián JJ. Realización de un seminario práctico de toma de decisiones obstétricas con formato de concurso por equipos. Experiencia piloto con estudiantes de Medicina. FEM, 2019, 22(4), 165-168. URL: https://www.educacionmedica.net/pdf/revista/2204/FEM 22041650251 9016 Pinel.pdf (ultimo acceso 29/9/20).

7. Álvarez Hernández J, Aguilar Parra JM, Fernández Campoy JM. Salguero García D, Pérez-Gallardo ER. El estrés ante los exámenes en los estudiantes universitarios. Propuesta de intervención. Int $J$ Develop Psychol, 2013, 2 (1), 179-187. URL: https://www.redalyc.org/pdf/3498/349852173008.pdf (último acceso 29/9/20).

8. Gual A, Palés-Argullós J. Una rara avis: la evaluación contínua. Educ Med, 2012, 15 (4), 185-188. URL: https://www.educacionmedica.net/pdf/revista/ 1504/1504 0185 0188.pdf (último acceso 29/9/20). 